\title{
Radiation therapy for locoregionally advanced nasopharyngeal carcinoma in elderly patients
}

\author{
Qiaojuan Guo • Weiping Jiang • Shaojun Lin • \\ Ling Yang • Chuanben Chen • Luying Xu • \\ Junxin Wu Jianji Pan
}

Received: 6 September 2012 / Accepted: 11 September 2012 /Published online: 30 September 2012

(C) Springer-Verlag Berlin Heidelberg 2012

\begin{abstract}
Objective The aim of this study was to evaluate the treatment outcome and toxicities of radiation therapy alone for elderly patients with locoregionally advanced nasopharyngeal carcinoma (NPC).

Methods Seventy-three patients of 70 years old or above treated at our institution between January 1995 and December 2008 for locoregionally advanced NPC (stage III-IVA) were reviewed and analyzed. All patients were treated in a uniform fashion using our institutional protocol with using conformal radiation therapy alone. Measured outcomes vival (TSS), disease-free survival (DFS), local control (LC), regional relapse-free survival (RRFS), distant metastasis free survival (DMFS), as well as late toxicity.

Results The median follow-up for all 73 patients at the time of this analysis was 45 months (range, 5-152 months). The 4-year OS, TSS, DFS, LC, RRFS, and DMFS were 51.0, $64.5,54.7,83.7,80.6$, and 63.5, respectively. Radiation therapy was well tolerated in this group of patients. Multivariate analyses revealed that clinical stage $(P=0.006)$ and comorbidities $(P=0.018)$ were significant prognosticators for OS, both $\mathrm{N}$ classification $(P=0.023)$ and comorbidities

Q. Guo $\cdot$ W. Jiang $\cdot$ S. Lin $\cdot$ L. Yang $\cdot$ C. Chen $\cdot$ L. Xu

J. Wu $(\bowtie) \cdot J$. Pan $(\bowtie)$

Department of Radiation Oncology,

Cancer Hospital of Fujian Medical University,

No. 420 Fuma Road,

Fuzhou, 350014, China

e-mail: junxinwu@126.com

e-mail: panjianji@126.com

\section{W. Jiang}

Department of Radiation Oncology,

Zhongshan Hospital of Xiamen University,

No. 201-209, South Hubin Road,

Xiamen, 361004, China
\end{abstract} included 4-year overall survival (OS), tumor-specific sur-
$(P=0.032)$ were significant prognosticators for DFS, N classification was the only independent prognostic factor for both TSS $(P=0.002)$ and DMFS $(P=0.004)$; and residual disease in the primary site was the only independent predictive factor for LC $(P=0.026)$.

Conclusion Radiation therapy alone can achieve a reasonable local and regional control in NPC patients with advanced age; however, distant metastasis remained the dominant failure pattern of our series. The presence of comorbidities was an important negative prognostic factor for treatment outcome.

Keywords Nasopharyngeal carcinoma · Elderly patients . Radiotherapy $\cdot$ Long-term efficacy

\section{Introduction}

Radiation therapy is the mainstay treatment for patients who have nonmetastatic nasopharyngeal carcinoma (NPC). As the results for radiation therapy alone for NPC patients with locoregionally advanced disease are suboptimal [1,2], patients of this subgroup are generally treated with combined chemoradiation therapy [3]. Local control, disease-free, and overall survival rates of $67-80,58-72$, and $67-80 \%$ have been reported for stage IIB-IVB NPC treated with this combined strategy using concurrent chemotherapy and conventional radiation [4-9]. However, the addition of chemotherapy to radiation therapy is potentially hazardous, and the adverse effects induced by concurrent chemotherapy are substantial.

Treatment-induced adverse effects may be of particular importance to patients with advanced age who are more likely to suffer from impaired general health status due to poorer tolerability to severe reactions as well as concurrent comorbidities. For this group of patients with locoregionally 
advanced NPC, definitive therapy may be limited to radiotherapy alone. Although outcome after radiation therapy alone for NPC has been repeatedly reported, the currently available relevant literature is limited for locoregionally advanced NPC patients older than 70 years of age.

The aim of this report is to document the outcome of a relatively large group of patients with advanced age with locoregionally advanced NPC, treated in a uniform fashion at the Cancer Hospital of Fujian Medical University, China, with conventional radiotherapy without any form of chemotherapy. Predictive factors for prognosis especially survival and treatment complications of this group of patients were also analyzed.

\section{Methods}

Patients' characteristics and evaluation

Between January 1995 and December 2008, 109 consecutive and nonselected patients of age with 70 or older were treated by conventional radiation therapy alone at our institution. All patients had pathologically confirmed poorly differentiated or undifferentiated carcinomas, which were originally staged according to the Chinese 1992 staging system [10]. Eighty-four patients were diagnosed with locoregionally advanced disease (i.e., stage III-IVA) and were considered not suitable to receive any types of chemotherapy by their attending radiation oncologists due to substantial comorbidities or declined chemotherapy.

Pretreatment evaluation consisted of a complete history and physical examination, direct nasopharyngeal endoscopy, biopsy of the nasopharynx, complete blood count, liver function tests, chest X-ray, CT scan of the head and neck, ultrasound of the liver, total-body bone scan, and dental evaluation. A total of 73 patients were included in this analysis, and 11 patients were excluded for the following reasons: one patient were noticed to have liver metastasis when he had just finished his treatment, two patients were lost to follow-up after the completion of radiation therapy (RT), and the remaining eight patients received radiation treatment deviated from our institutional protocol (e.g., used preauricular field alone for the boost).

Significant comorbidities were observed in 28 of the 73 patients $(38.36 \%)$. Comorbidities observed including gastric cancer, rectal cancer, hypopharyngeal cancer, hypertension, diabetes, cerebrovascular disease, coronary disease, thyroid diseases, fatty liver syndrome, bronchiectasis, bronchitis, and emphysema. Patients' characteristics are listed in Table 1.

\section{Radiation therapy}

All patients were treated with definitive radiation alone without any form of chemotherapy according to our
Table 1 Demographic and clinical characteristics of cohort $(n=73)$

\begin{tabular}{|c|c|c|}
\hline Characteristic & Number & Percent \\
\hline \multicolumn{3}{|l|}{ Gender } \\
\hline Male & 59 & 80.82 \\
\hline Female & 14 & 19.18 \\
\hline \multicolumn{3}{|c|}{ Treatment break } \\
\hline Yes & 13 & 17.81 \\
\hline No & 60 & 82.19 \\
\hline \multicolumn{3}{|l|}{ Clinical stage } \\
\hline III & 39 & 53.43 \\
\hline IV & 34 & 46.57 \\
\hline \multicolumn{3}{|l|}{$\mathrm{T}$ classification } \\
\hline $\mathrm{T} 1$ & 4 & 5.48 \\
\hline $\mathrm{T} 2$ & 15 & 20.55 \\
\hline $\mathrm{T} 3$ & 18 & 24.66 \\
\hline $\mathrm{T} 4$ & 36 & 49.32 \\
\hline \multicolumn{3}{|c|}{$\mathrm{N}$ classification } \\
\hline No & 14 & 19.18 \\
\hline N1 & 28 & 38.36 \\
\hline $\mathrm{N} 2$ & 29 & 39.72 \\
\hline $\mathrm{N} 3$ & 2 & 2.74 \\
\hline \multicolumn{3}{|c|}{ Residual in primary site } \\
\hline No & 55 & 75.34 \\
\hline Yes & 18 & 24.66 \\
\hline \multicolumn{3}{|c|}{ Residual in neck } \\
\hline No & 51 & 69.86 \\
\hline Yes & 22 & 30.14 \\
\hline \multicolumn{3}{|l|}{ Comorbidity } \\
\hline Yes & 46 & 63.01 \\
\hline No & 27 & 36.99 \\
\hline
\end{tabular}

institutional protocol for elderly NPC patients. Patients were simulated using conventional simulation and treated supine with a customized head shell. Phase 1 of RT consisted of two large parallel-opposed lateral facio-cervical fields to 36-40 Gy. These fields were then reduced to off-cord with posterior electron matching fields to a total of $50 \mathrm{~Gy}$. The nasopharynx would receive a total of 70-72 Gy after an anterior and two lateral fields boost. Initial dose and treatment techniques for the neck in both groups of patients with or without neck adenopathy were identical: node-negative patients would receive 50 Gy to the neck and node-positive patients would receive an additional boost to a total dose of 66-70 Gy to involved region(s). Radiation was delivered at 2-Gy daily fractions, five times a week.

Follow-up

The first clinical assessment of local disease using nasoendoscopy was typically at the end of radiation therapy. All patients were followed up clinically by an otolaryngologist 
and/or radiation oncologist every 2 months in the first year after completion of radiation, every 3 months in the second year, every 4 months in the third year, every 6 months in the fourth and fifth years, and annually thereafter. Diagnosis of local and regional recurrence was made histologically. Distant metastases were diagnosed either by pathological or radiological evidence. Treatment-related toxicities were accessed and scored according to the RTOG radiation morbidity scoring criteria at each follow-up [11].

\section{Data analysis}

Data were analyzed using SPSS version 16.0 (SPSS Inc., Chicago, USA). Overall survival (OS), tumor-specific survival (TSS), disease-free survival (DFS), local control (LC), regional relapse-free survival (RRFS), and distant-metastasis free survival (DMFS) rates were calculated by the KaplanMeier method. The duration of OS was measured from diagnosis until death or date of the last follow-up visit for patients still alive. TSS interval was calculated from the date of diagnosis to time of death caused by tumor progression or treatment complications. Log-rank test was used to detect differences in survival between different groups. Multivariate analysis using the Cox proportional hazard model was performed for survival to identify independent predictors among various potential prognostic factors, including gender, $\mathrm{T}$ classification, $\mathrm{N}$ classification, clinical stage, radiation interrupted or not, residual disease in the primary site or neck, and comorbidities. A two-sided $P$ value of $<0.05$ was considered to be statistically significant.

\section{Results}

The median follow-up for all 73 patients at the time of this analysis was 45 months (range, 5-152 months).

Treatment response and compliance

Most of the patients could complete RT without a treatment break, and only $13(17.81 \%)$ patients required a break of more than 1 week mainly because of severe acute toxicities (such as mucositis and dermatitis). At the completion of RT, 18 patients $(24.66 \%)$ had residual diseases in the primary site, $22(30.14 \%)$ had residual diseases in the neck, and 5 $(6.85 \%)$ had residual disease in both the primary site and the neck nodal regions.

\section{Survival}

At the time of this analysis, 47 patients were deceased: 26 patients died from the effects of tumor progression, 3 patients died of severe late complications, and 16 patients died of other disease including gastric cancer, cardiac failure, cerebrovascular disease, and pulmonary infection. The causes of death of two additional cases were unknown.

OS, TSS, and DFS of the whole cohort are presented in Figs. 1, 2, and 3. The estimated 4-year OS, TSS, and DFS were $51.0,64.5$, and $54.7 \%$, respectively. Univariate analysis revealed that $\mathrm{N}$ classification, clinical stage, and comorbidities were potential prognostic factors for OS. However, only clinical stage and comorbidities were the significant independent factors for OS in multivariate analysis $(P=0.006$ for clinical stage and $P=0.018$ for comorbidity, Table 2, Fig. 1a, b). In addition, both $\mathrm{N}$ classification and comorbidities were identified as the significant predicting factors for DFS ( $P=0.023$ for $\mathrm{N}$ classification and $P=0.032$ for comorbidity, Table 2). N category was found to be the only independent prognostic factor for TSS in both univariate and multivariate analysis $(P=0.002$, Table 2$)$

\section{Local and regional control}

For the 18 patients who had residual disease at the end of treatment, CT/magnetic resonance imaging (MRI) scans revealed that the viable appearing disease was all within the radiation field. Five of these patients received a radiation boost to a total dose of 76-78 Gy at the primary site and were free of disease at the primary tumor by the last followup or death; for those who did not accept a boost, five patients developed local recurrence at a median time of 27 months (range, 15-56 months). At the time of censorship, a total of ten patients (including five patients who have residual disease at the end of treatment) presented with local recurrence (median, 23.5; range, 10-52 months). The estimated local control rate at 4 years is $83.7 \%$ for all 73 patients (Fig. 4). N classification and residual disease in primary site were noted to be the potential predictors for LC using log-rank test; however, only the later factor was identified to be significant in the Cox model $(P=0.026$, Table 2).

For the 22 patients ( $30.14 \%)$ who had neck node residual disease at the end of radiation, eight of them received a radiation boost at the lymph node to a total dose of 72 $85 \mathrm{~Gy}$ (median, 76 Gy), all maintained to be disease free at the site of lymph node till the last follow-up except for one patient. Seven patients experienced neck node dissection and were free of disease at the last visit; another seven patients were also offered, but refused, attempted salvage neck dissection or radiation boost and then manifested regional failure or distant metastases several months after the diagnosis of nodal relapse. Regional recurrence occurred in 11 patients; the estimated regional control rate at 4 years is $80.6 \%$ (Fig. 5). Prognostic analysis indicated that $\mathrm{T}$ classification, $\mathrm{N}$ classification, and residual disease in the neck were the potential prognostic factors found in 
Fig. 1 Overall survival for a clinical stage and $\mathbf{b}$ comorbidity of the whole cohort a

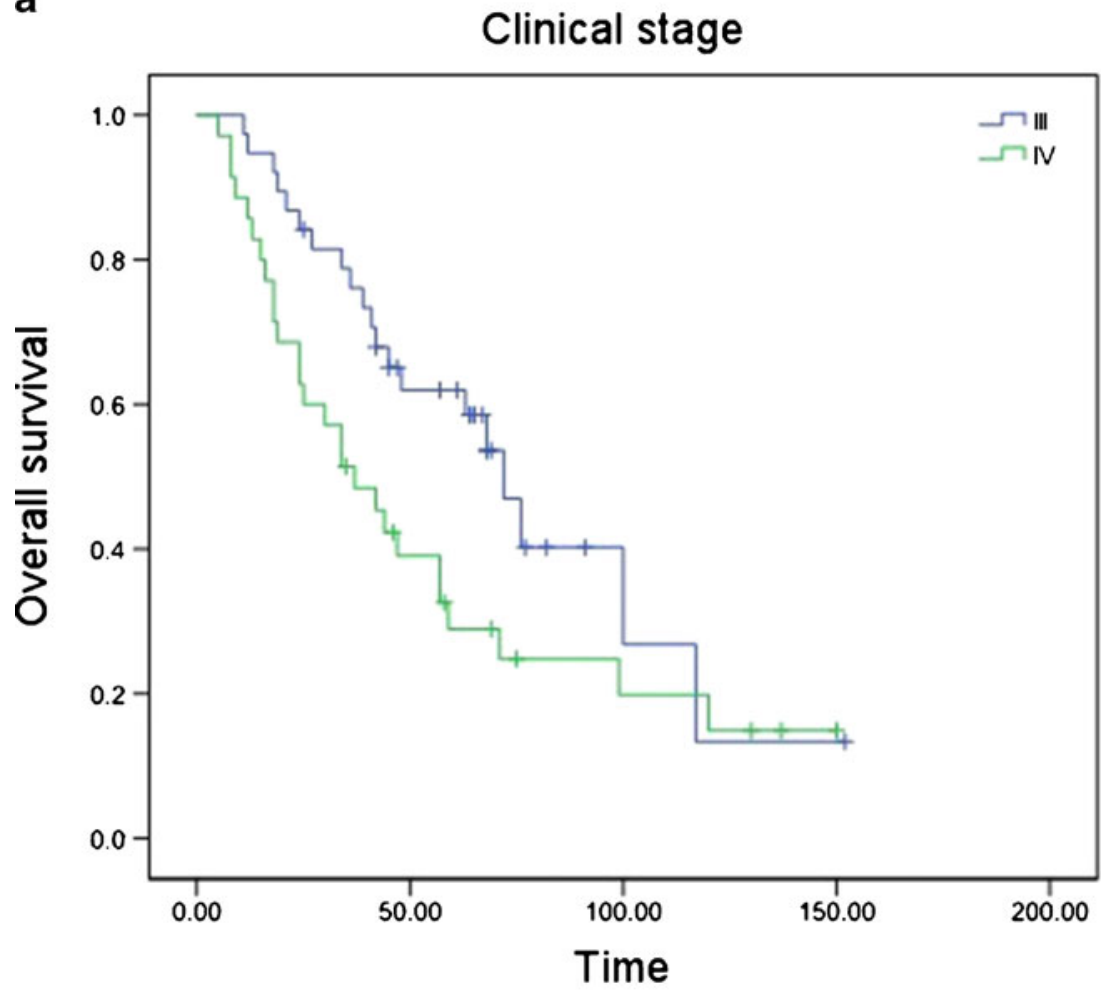

b

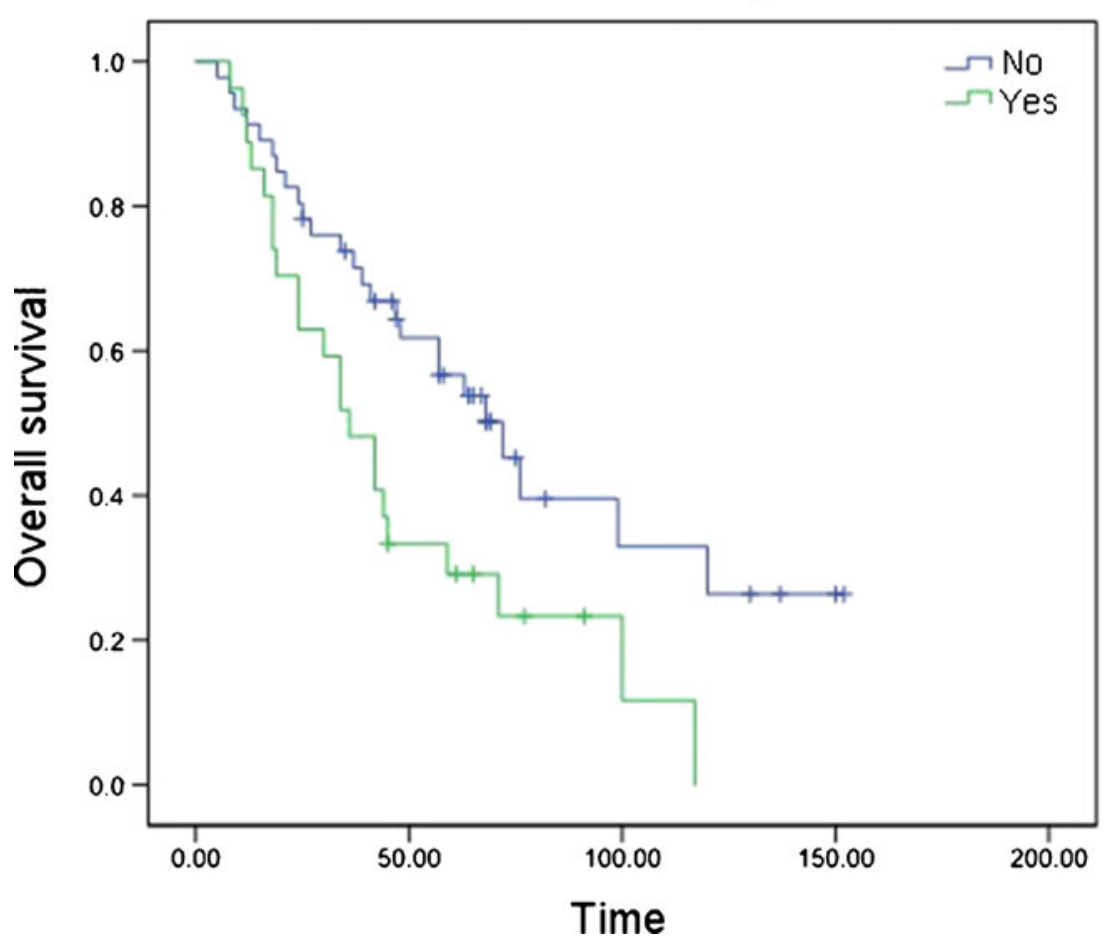

univariate analysis; however, none of them were significant in predicting regional control after multivariate analysis $(P=$ 0.816 for $\mathrm{T}$ classification, 0.465 for $\mathrm{N}$ classification, and 0.057 for residual disease in the neck, Table 2).
Distant metastasis

Twenty-five patients developed distant metastasis (DM), with a median time to distant metastasis at 18 months 


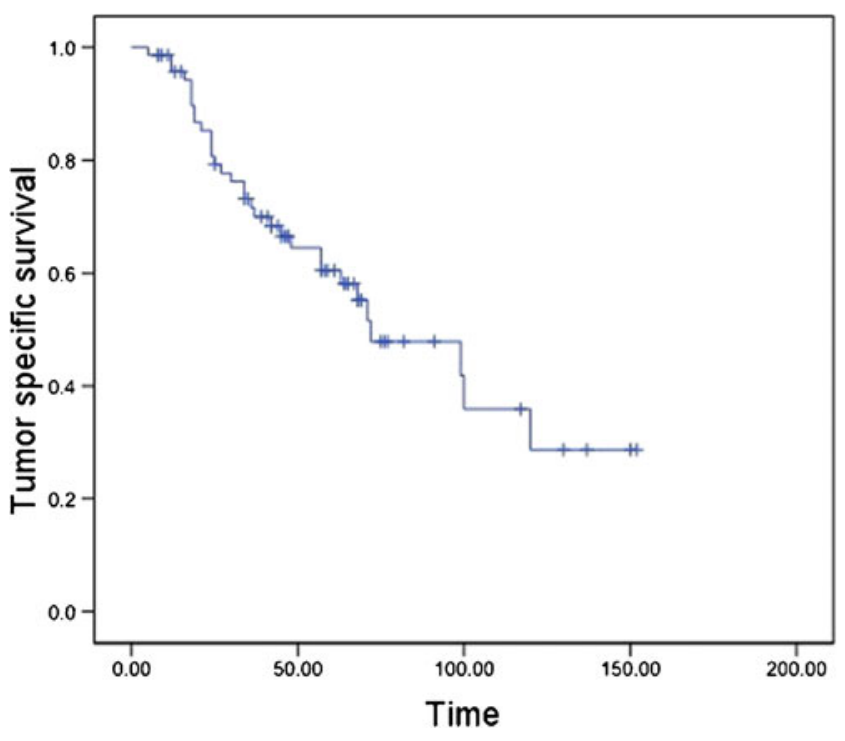

Fig. 2 Tumor-specific survival of the whole cohort

(range, 3-66). Most of the patients (76\%) developed DM within 2 years after definite radiotherapy. The most common sites of DM were bone, lung, liver, and brain, either single or multiple metastases, with the 4-year DMFS rate of $63.5 \%$ (Fig. 6). Prognostic analysis revealed that only $\mathrm{N}$ category was the significant predictor for DM $(P=0.004)$.

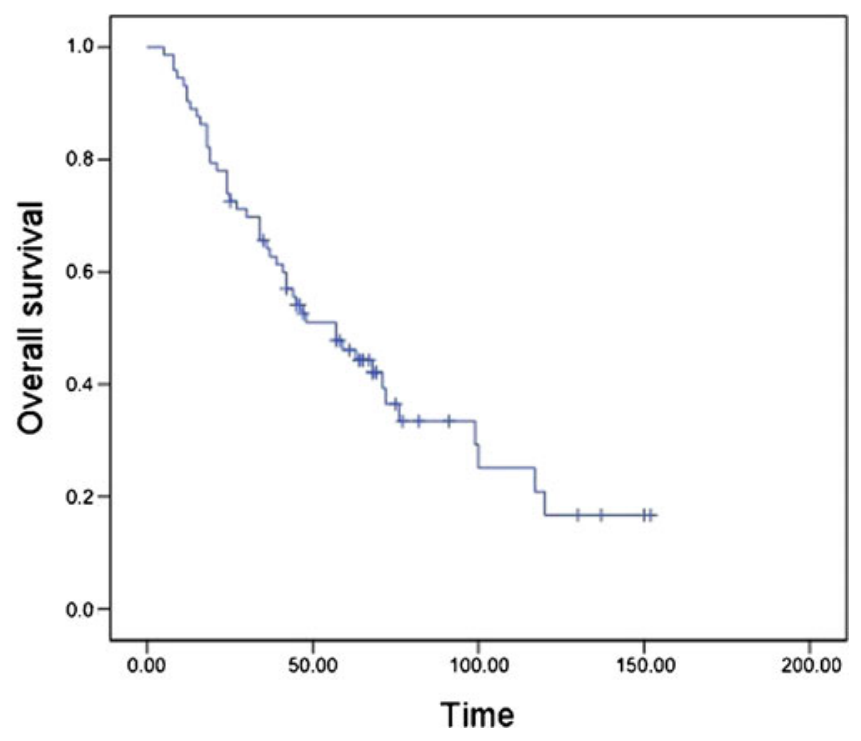

Acute and late adverse effects

Patients typically experienced the common acute and late toxicity of EBRT, including xerostomia, skin reaction, oral and pharyngeal mucositis, hearing deficit, olfactory change, and/or decreased taste, etc. Most of the patients tolerated the treatment well, but 13 patients suspended their treatment for
Fig. 3 Disease-free survival of the whole cohort

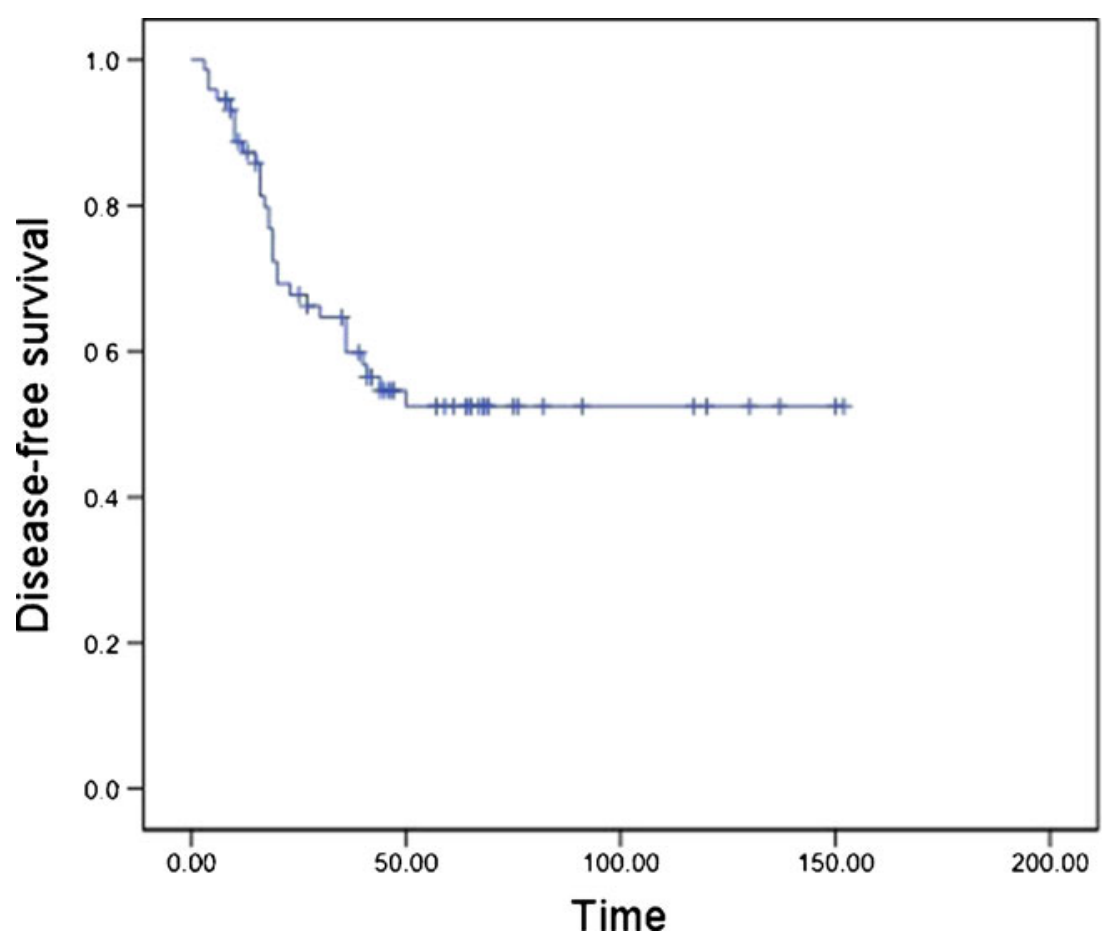


Table 2 Multivariate analysis of potential prognostic factors

\begin{tabular}{|c|c|c|c|c|c|c|c|c|c|}
\hline \multirow[t]{2}{*}{ Endpoint } & \multirow[t]{2}{*}{ Factor } & \multirow[t]{2}{*}{ B } & \multirow[t]{2}{*}{ SE } & \multirow[t]{2}{*}{ Wald } & \multirow[t]{2}{*}{$D f$} & \multirow[t]{2}{*}{ Sig } & \multirow[t]{2}{*}{$\operatorname{Exp}(B)$} & \multicolumn{2}{|c|}{$95 \%$ CI $\operatorname{Exp}(\mathrm{B})$} \\
\hline & & & & & & & & Lower & Upper \\
\hline \multirow[t]{3}{*}{ OS } & Clinical stage & 0.876 & 0.320 & 7.509 & 1 & 0.006 & 2.401 & 1.283 & 4.493 \\
\hline & $\mathrm{N}$ classification & 0.390 & 0.214 & 3.311 & 1 & 0.069 & 1.477 & 0.970 & 2.247 \\
\hline & Comorbidity & 0.722 & 0.305 & 5.618 & 1 & 0.018 & 2.058 & 1.133 & 3.738 \\
\hline \multirow[t]{2}{*}{ LC } & $\mathrm{N}$ classification & 0.288 & 0.400 & 0.517 & 1 & 0.472 & 1.334 & 0.608 & 2.924 \\
\hline & RPS & 1.434 & 0.645 & 4.944 & 1 & 0.026 & 4.194 & 1.185 & 14.841 \\
\hline \multirow[t]{3}{*}{ RRFS } & $\mathrm{N}$ classification & 0.390 & 0.535 & 0.533 & 1 & 0.465 & 1.478 & 0.518 & 4.214 \\
\hline & $\mathrm{T}$ classification & 0.073 & 0.315 & 0.054 & 1 & 0.816 & 0.929 & 0.502 & 1.722 \\
\hline & $\mathrm{RN}$ & 1.301 & 0.682 & 3.636 & 1 & 0.057 & 3.673 & 0.964 & 13.993 \\
\hline \multirow[t]{2}{*}{ DFS } & Comorbidity & 0.779 & 0.363 & 4.620 & 1 & 0.032 & 2.180 & 1.071 & 4.438 \\
\hline & $\mathrm{N}$ classification & 0.564 & 0.248 & 5.194 & 1 & 0.023 & 1.758 & 1.082 & 2.857 \\
\hline
\end{tabular}

$O S$ overall survival, $L C$ local control, RRFS regional relapsefree survival, $D F S$, disease-free survival, $R P S$ residual in primary site, $R N$ residual in neck diagnosed in their advanced age (i.e., $\geq 70$ years old). Although concurrent chemoradiation therapy is considered as the standard treatment strategy for patients with locoregionally advanced NPC, patients with advanced age, particularly those with concurrent impaired health condition, are not suitable to this aggressive regimen. In this study, we reviewed a group of patients over 70 years old diagnosed with stage III-IVA undifferentiated or poor differentiated NPC who were treated with definitive radiation therapy alone using conventional technique. Our results demonstrated that the 4-year OS, TSS, DFS, LC, RRFS, and DMFS were $51.0,64.5,54.7,83.7,80.6$, and $63.5 \%$, respectively. Radiation therapy was well tolerated in this group of patients. Multivariate analyses for prognostic indicators in Southern China, and a substantial portion of patients were

Fig. 4 Local control rate at 4 years

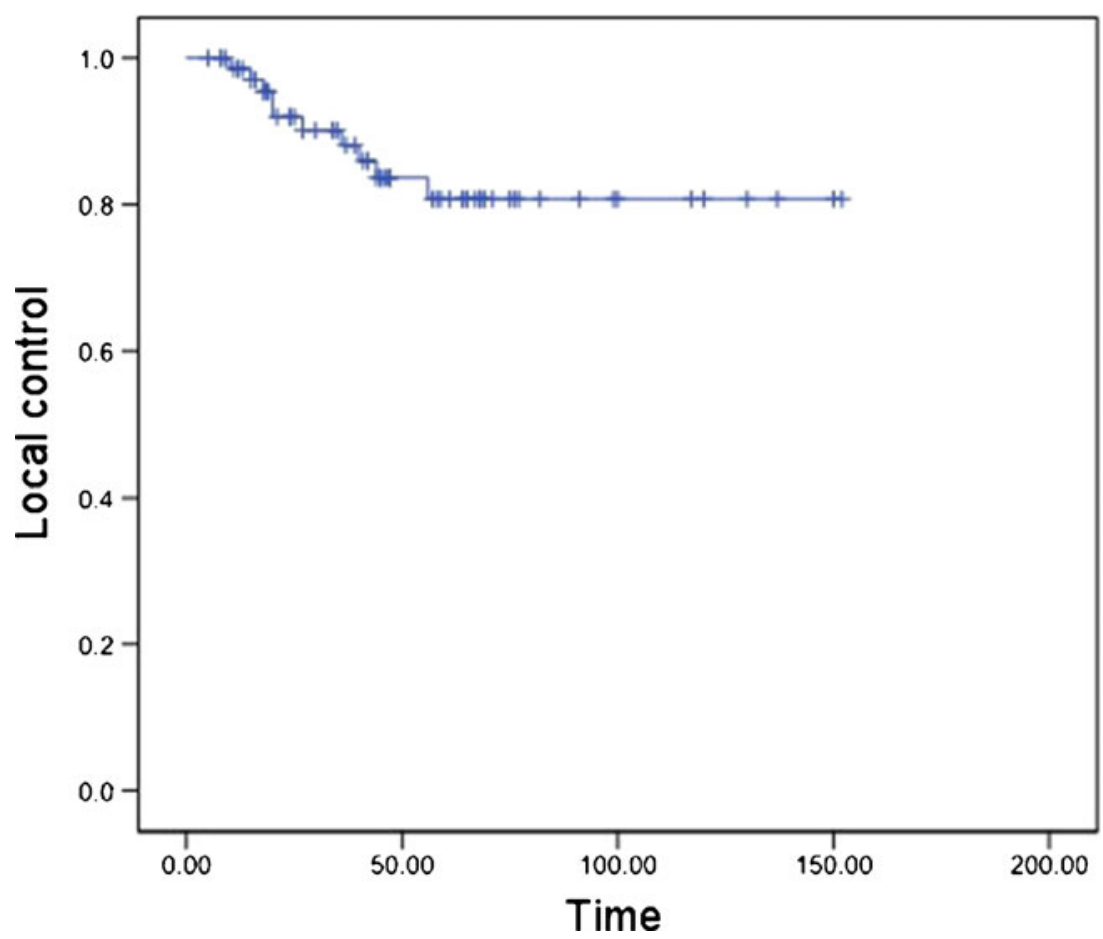


Fig. 5 Regional relapse-free survival of the whole cohort

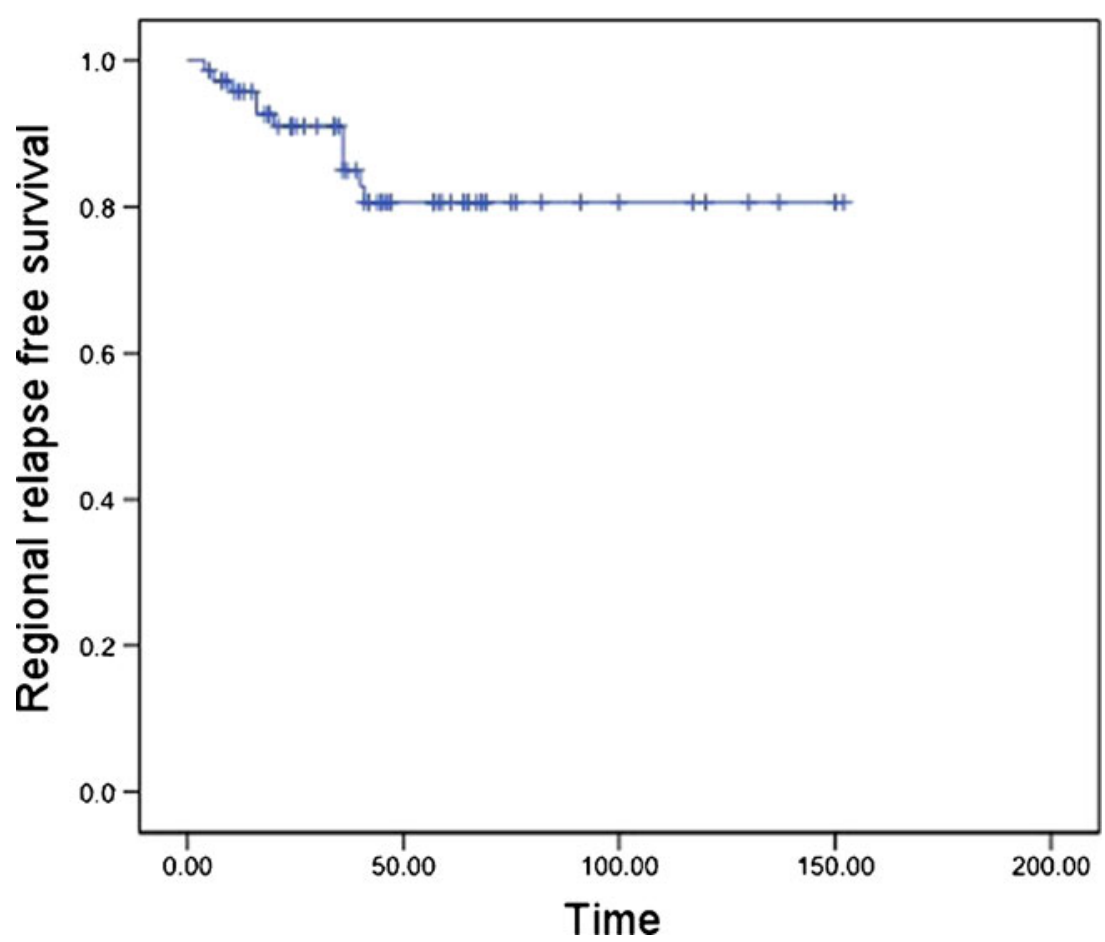

revealed that clinical stage and comorbidities were the significant prognostic factors for OS. For DFS, both N classification and comorbidities were found to be significant, and $\mathrm{N}$ category was identified as the independent prognostic factor for both TSS and DMFS, residual disease in primary site was found to be the independent predicting factor for LC (Table 2).
An increasing incidence of NPC was observed in elderly patients in China [12,13]. Moreover, a second peak of NPC risk by ages $65-79$ years had also been discovered emerging across the low-risk populations [14]. Our experience and results presented are of particular importance that we represent the first report to address the treatment outcome for aged patients (i.e., $\geq 70$ years old) with locoregionally
Fig. 6 Distant metastasis-free survival of the whole cohort

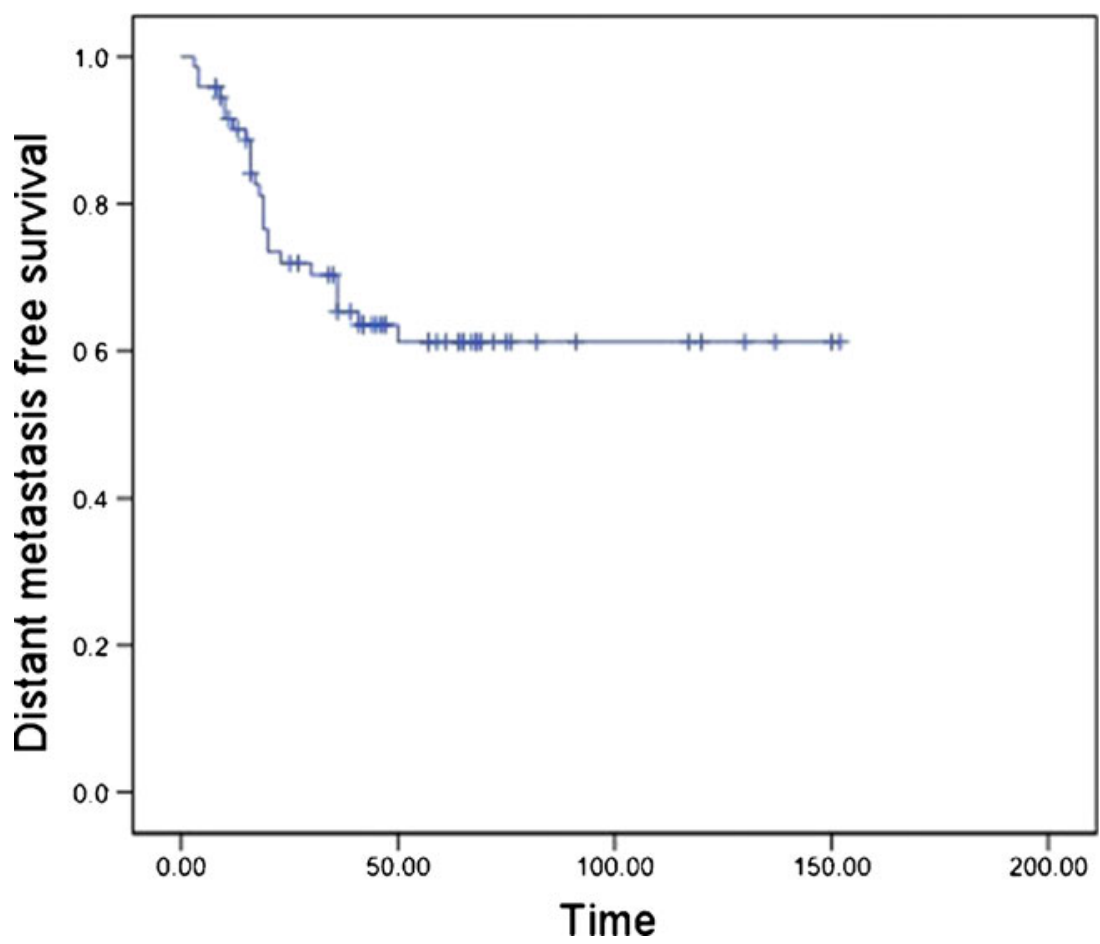


Table 3 Cumulative incidence of symptomatic late complications $(n=73)$

\begin{tabular}{llr}
\hline Complications & Number & Percent \\
\hline ZGrade 2 trismus & 6 & 8.22 \\
ZGrade 2 neck fibrosis & 13 & 17.81 \\
Cranial neuropathy & 3 & 4.11 \\
Temporal lobe necrosis & 2 & 2.74 \\
Nasopharyngeal ulceration & 1 & 1.37 \\
\hline
\end{tabular}

advanced stage who underwent radiation therapy alone; it may be a reasonable choice for this subgroup of patients who often present with concurrent morbidities, thus impaired health condition, and who are not suitable to receive aggressive regimen (i.e., combined chemoradiation therapy). Radiation therapy alone may induce relatively lower incidence of complication while providing an acceptable good treatment results.

Numerous published papers have reported the treatment outcome of radiation therapy alone on locoregionally advanced NPC patients. Most of them demonstrated a 5-year OS of over $50 \%$ for poorly or undifferentiated diseases. The experience from Ma et al. [15] revealed that their 5-year OS and relapse-free survival for stage III and stage IV patients were 53 vs. 37 and 51 vs. $36 \%$, respectively. Chan et al. [6, 9] from Hong Kong reported their 5-year OS and PFS of 58.6 and $52.1 \%$, respectively. Another set of data from Hong Kong reported by Chua et al. [16] suggested a 5 -year OS of $58.1 \%$ and 5-year TSS, LRRFS, and DMFS rates of 58.1, 59.5, and $70 \%$, respectively. This is a pool data analysis that included one of their previous study [17] and that reported by Ma et al. [18]. Similar results were reported by Lin et al. [5] from Taiwan. With a relatively short follow-up time, Wee et al. [19] from Singapore demonstrated that the 3-year OS and DFS were 65 and $63 \%$, respectively. Ten-year results have been reported by Johansen et al. [20] in Denmark and Yi et al. [2] in China; data from Denmark revealed that their OS of this subgroup was 49 and $27 \%$ for stage III and stage VI, respectively, and that from China demonstrated their 5- and 10-year OS, DFS, and LC of 71.3 and 66.5, 52.2 and 52.1, 81.2 and $76.7 \%$, respectively.

The OS rate of the current study was understandably less optimal than those mentioned above, although we had a lower proportion of stage IV (45.21\%), when compared to other studies, such as those of Yi et al. [2] (62.07\%), Lin et al. [5] (84.62 \%), Johansen et al. [20] (68.46\%), and Wee et al. [19] (52 \%). This suggested that survival outcome of elderly patients (i.e., $\geq 70$ years old) with locoregionally advanced stage was inferior to those with relatively young age, further confirming that age is a negative prognostic factor for NPC patients. Our experience previously published has confirmed the significance of age as an adverse prognostic factor [21]. This subgroup is more often associated with a variety of declining physiological functions, multiple comorbidities, and reduced organ reserve on account of advanced aging, which might lead to a detrimental effect on ability to withstand radiation toxicities and worse outcome. For the 47 patients who died, almost 1/3 of them were contributed to comorbidities instead of NPC, and $46(63.01 \%)$ patients in our study had comorbidities. As shown in the prognostic analysis, comorbidities are a significant predicting factor for OS and DFS. However, it is not a significant prognosticator for TSS. Notably, varied staging systems used in these trials may play a role in the discrepancy of the results; the Chinese 1992 staging system was used in our series and that of Yi et al. [2], and the Johansen study [20] used the 1982 UICC system, whereas all other studies mentioned above used the 1997 AJCC system [5, 9, 16, 19]. However, if this system was used to reclassify our patients, some of them would be downstaged and excluded from our series; the survival results may be worse.

It is important to mention and compare our data with the results of the pivotal study of the Intergroup 0099 reported by Al-Saraff et al., which randomly compared concurrent chemoradiation with radiation alone using a conventional technique[ 4,8$]$. The reported survival outcome was substantially lower than that reported in our series and other series from endemic areas mentioned above. The 3-year OS, DFS, LRRFS, and DMFS reported were 47, 24, 59, and $65 \%$, respectively. In 2001, the final update of the Intergroup 0099 trial showed a 5-year OS and DFS of 37 and $29 \%$, respectively. One explanation was that more than a quarter of the patients in the Intergroup trial had the keratinizing type (WHO type I) of NPC, whereas more than $90 \%$ of patients in endemic areas have the nonkeratinizing type (mainly WHO type III). It has been demonstrated that the prognosis for the former is significantly poorer than that for the latter [22-24]. Another possible reason may be that the Intergroup trial had a higher proportion $(>90 \%)$ of patients with nonmetastatic stage IV disease (1992 AJCC staging system), when compared to a lower proportion of $45.21 \%$ in our series. Moreover, the different staging systems could further explain, at least in part, the discrepancy of the results.

Both the 4-year LC and RRFS of the present study were indicated to be over $80 \%$, suggesting that a reasonable disease control could be achieved in this subgroup of patients. For the patients who had residual disease, either at the primary site or neck, all but one of the patients who received salvage treatment were free of disease at the time of censorship. And residual disease in primary site was found to be the significant independent factor governing LC. Although residual disease in the neck was not identified as the significant predicting factor in the Cox model, univariate analysis showed that patients with residual disease in the neck had much lower regional control (89.6 vs. $59.2 \%$, $P=0.005$ ). Our results suggested that a boost dose for a 
pathologically positive residual lesion in the nasopharynx is necessary, as has proven by other studies [2, 25]. In addition, selective neck dissection also has been demonstrated to be important to the patients who have persistent cervical lymph node at the end of treatment, as they may have a longer survival time and better prognosis [26]. Radiation boost for the patients with residual disease in the neck is also an effective method, as regional recurrence occurred in only one of the eight patients who received a radiation boost in our cohort at the time of censorship. However, salvage treatment by radiation boost may be associated with treatment-related morbidity, secondary to radiation-induced injury, and the tolerance of elderly patients has been a major clinical issue. Various modern external beam radiation techniques, such as stereotactic radiosurgery [27, 28], and intensity-modulated radiation therapy (IMRT) [29] have been utilized to achieve dose escalation with some success. We believe that, with the help of these modern radiotherapy techniques, escalation of the dose may be easily achieved without increasing treatment-related late complication of elderly patients.

Several limitations and pitfalls of our studies should be addressed. First, most of the patients were staged primarily by CT scans, which have shown lower accuracy as compared with MRI. This may pose some uncertainties in the staging of our patients and, thus, may have an impact on the results. In addition, due to the limitation of the Chinese 1992 staging system [30], the prognostic significance of clinical stage, $\mathrm{T}$, and $\mathrm{N}$ category may need to be further demonstrated. Moreover, some of the late toxicities for elderly patients in this study may have been underestimated, because many of them might have died before the long-term toxicities could develop. For example, the median period of cranial nerve palsy approached 8 years, as reported by Kong et al. [31].

\section{Conclusion}

Radiation therapy alone can achieve a reasonable local and regional control in elderly patients and is a reasonable choice for this subgroup of patients. Distant metastasis remained to be the dominant failure pattern of our series. Whether modern radiation techniques such as IMRT alone or the combination of targeted therapy with radiation therapy could bring a survival and adverse effect benefit for the elder NPC patients requires further investigation.

Conflict of interest The authors declare that they have no conflict of interest.

\section{References}

1. Yeh SA, Tang Y, Lui CC et al (2005) Treatment outcomes and late complications of 849 patients with nasopharyngeal carcinoma treated with radiotherapy alone. Int J Radiat Oncol Biol Phys 62:672-679

2. Yi JL, Gao L, Huang XD et al (2006) Nasopharyngeal carcinoma treated by radical radiotherapy alone: ten-year experience of a single institution. Int J Radiat Oncol Biol Phys 65:161-168

3. Lee A, Ng W, Chan O et al (2012) If concurrent-adjuvant chemoradiotherapy is beneficial for locoregionally advanced nasopharyngeal carcinoma, would changing the sequence to inductionconcurrent achieve better outcome? J Radiat Oncol 1:107-115

4. Al-Sarraf M, LeBlanc M, Giri PG et al (1998) Chemoradiotherapy versus radiotherapy in patients with advanced nasopharyngeal cancer. Phase III randomized Intergroup study 0099. J Clin Oncol 16:1310-1317

5. Lin JC, Jan JS, Hsu CY et al (2003) Phase III study of concurrent chemoradiotherapy versus radiotherapy alone for advanced nasopharyngeal carcinoma. Positive effect on overall and progressionfree survival. J Clin Oncol 21:631-637

6. Chan AT, Teo PM, Ngan RK et al (2002) Concurrent chemotherapyradiotherapy compared with radiotherapy alone in locoregionally advanced nasopharyngeal carcinoma. Progression-free survival analysis of a phase III randomized trial. J Clin Oncol 20: 2038-2044

7. Lee AW, Lau WH, Tung SY et al (2005) Preliminary results of a randomized study on therapeutic gain by concurrent chemotherapy for regionally-advanced nasopharyngeal carcinoma. NPC-9901 Trial by the Hong Kong Nasopharyngeal Cancer Study Group. J Clin Oncol 23:6966-6975

8. Al-Sarraf M, LeBlanc M, Giri PG et al (2001) Superiority of fiveyear survival with chemoradiotherapy versus radiotherapy in patients with locally advanced nasopharyngeal cancer. Intergroup (0099), SWOG 8892, RTOG 8817, ECOG 2388 Phase III study: final report. Proc Am Soc Clin Oncol 20:227a

9. Chan AT, Leung SF, Ngan RK et al (2005) Overall survival after concurrent cisplatin-radiotherapy compared with radiotherapy alone in locoregionally advanced nasopharyngeal carcinoma. J Natl Cancer Inst 97:536-539

10. Min HQ, Hong MH, Ma J et al (1994) A new staging system for nasopharyngeal carcinoma in China. Int J Radiat Oncol Biol Phys 30:1037-1042

11. Cox JD, Stetz J, Pajak TF et al (1995) Toxicity criteria of the Radiation Therapy Oncology Group (RTOG) and the European Organization for Research and Treatment of Cancer (EORTC). Int J Radiat Oncol Biol Phys 31:1341-1346

12. Jia WH, Huang QH, Liao J et al (2006) Trends in incidence and mortality of nasopharyngeal carcinoma over a 20-25 year period (1978/1983-2002) in Sihui and Cangwu counties in southern China. BMC Cancer 6:178

13. Wei KR, Yu YL, Yang YY et al (2010) Epidemiological trends of nasopharyngeal carcinoma in China. Asian Pac J Cancer Prev 11:29-32

14. Bray F, Haugen M, Moger TA et al (2008) Age-incidence curves of nasopharyngeal carcinoma worldwide: bimodality in low-risk populations and aetiologic implications. Cancer Epidemiol Biomarkers Prev 17:2356-2365

15. Ma J, Mai HQ, Hong MH et al (2001) Is the 1997 AJCC staging system for nasopharyngeal carcinoma prognostically useful for Chinese patient populations? Int J Radiat Oncol Biol Phys 50:11811189

16. Chua DT, Ma J, Sham JS et al (2005) Long-term survival after cisplatin-based induction chemotherapy and radiotherapy for nasopharyngeal carcinoma: a pooled data analysis of two phase III trials. J Clin Oncol 23:1118-1124 
17. Chua DTT, Sham JST, Choy D et al (1998) Preliminary report of the Asian-Oceanian Clinical Oncology Association randomized trial comparing cisplatin and epirubicin followed by radiotherapy versus radiotherapy alone in the treatment of patients with locoregionally advanced nasopharyngeal carcinoma. Cancer 83:2270-2283

18. Ma J, Mai H, Hong M et al (2001) Results of a prospective randomized trial comparing neoadjuvant chemotherapy plus radiotherapy with radiotherapy alone in patients with locoregionally advanced nasopharyngeal carcinoma. J Clin Oncol 19:1350-1357

19. Wee J, Tan EH, Tai BC et al (2005) Randomized trial of radiotherapy versus concurrent chemoradiotherapy followed by adjuvant chemotherapy in patients with American Joint Committee on Cancer/ International Union against cancer stage III and IV nasopharyngeal cancer of the endemic variety. J Clin Oncol 23:6730-6738

20. Johansen LV, Mestre M, Overgaard J (1992) Carcinoma of the nasopharynx. Analysis of treatment results in 167 consecutively admitted patients. Head Neck 14:200-207

21. Xu L, Pan J, Wu J et al (2010) Factors associated with overall survival in 1706 patients with nasopharyngeal carcinoma: significance of intensive neoadjuvant chemotherapy and radiation break. Radiother Oncol 96:94-99

22. Shanmugaratnam K, Chan SH, de-The G et al (1979) Histopathology of nasopharyngeal carcinoma: correlations with epidemiology, survival rates and other biological characteristics. Cancer 44:1029-1044

23. Hoppe RT, Williams J, Warnke R et al (1978) Carcinoma of the nasopharynx: the significance of histology. Int J Radiat Oncol Biol Phys 4:199-205
24. Neel HB 3rd, Taylor WF (1989) New staging system for nasopharyngeal carcinoma. Long-term outcome. Arch Otolaryngol Head Neck Surg 115:1293-1303

25. Yan JH, Xu GZ, Hu YH et al (1990) Management of local residual primary lesion of nasopharyngeal carcinoma. II. Results of prospective randomized trial on booster dose. Int J Radiat Oncol Biol Phys 18:295-298

26. Zhang L, Zhu YX, Wang Y et al (2011) Salvage surgery for neck residue or recurrence of nasopharyngeal carcinoma: a 10-year experience. Ann Surg Oncol 18:233-238

27. Siddiqui F, Kunos C, Paulino A (2012) Stereotactic body radiation therapy in head and neck, gynecologic, and pediatric malignancies. J Radiat Oncol 1:31-42

28. Hara W, Loo BW Jr, Goffinet DR et al (2008) Excellent local control with stereotactic radiotherapy boost after external beam radiotherapy in patients with nasopharyngeal carcinoma. Int $\mathrm{J}$ Radiat Oncol Biol Phys 71:393-400

29. Wang T, Riaz N, Cheng S et al (2012) Intensity-modulated radiation therapy for nasopharyngeal carcinoma: a review. J Radiat Oncol 1:129-146

30. Committee of Chinese Clinical Staging of Nasopharyngeal Carcinoma, (2009) Report on the revision of nasopharyngeal carcinoma '92 staging. Chin J Radiat Oncol 18:2-6 [in Chinese]

31. Kong L, Lu JJ, Liss AL et al (2011) Radiation-induced cranial nerve palsy: a cross-sectional study of nasopharyngeal cancer patients after definitive radiotherapy. Int J Radiat Oncol Biol Phys 79:1421-1427 